\title{
DESARROLLO E INNOVACIÓN EN EL CURRICULUM DEL PROGRAMA DE LA LICENCIATURA EN INGENIERÍA AGRÍCOLA DEL INSTITUTO TECNOLÓGICO DE COSTA RICA
}

\author{
Andrei Fëdorov Fëdorov ${ }^{1}$ \\ Asesor del Centro de Desarrollo Académico \\ del Instituto Tecnológico de Costa Rica. \\ Cartago, Costa Rica
}

Isabel Guzmán Arias ${ }^{2}$

Profesora y coordinadora de la comisión de rediseño de la Escuela de Ingeniería Agrícola del Instituto Tecnológico de Costa Rica

Cartago, Costa Rica

\begin{abstract}
Resumen: El presente artículo describe el panorama general del recién concluido proceso de rediseño curricular del plan de estudios de la licenciatura en Ingeniería Agrícola del Instituto Tecnológico de Costa Rica. A sus treinta años de existencia, este programa ha alcanzado su madurez; no obstante, ante las nuevas exigencias del desarrollo de la disciplina, del entorno productivo nacional e internacional y del contexto universitario y social, es necesario realizar su replanteamiento. El principal objetivo del proceso consiste en actualizar el programa de una manera integral y asi procurar una mayor calidad formativa en esta área. Se describen los antecedentes, la metodología y los logros más destacados del rediseño.
\end{abstract}

Palabras clave: Diseño curricular, Ingeniería Agrícola; Educación en ingeniería; Educación superior.

\section{Introducción}

Uno de los fines del Instituto Tecnológico de Costa Rica (ITCR o TEC), consiste en:

Formar profesionales en el campo tecnológico que aúnen al dominio de su disciplina una clara conciencia del contexto socioeconómico, cultural y ambiental en que la tecnología se genera, transfiere y aplica, lo cual les permita participar en forma crítica y creativa en las actividades productivas nacionales. (ITCR, 2005, p. 5)

La institución ha velado por el cumplimiento de este principio y en procura de ello, desde finales de la década de los noventa, ha ido estableciendo y tomando una serie de políticas y decisiones orientadas hacia el mejoramiento continuo y acreditación de la calidad de sus programas académicos (Fëdorov, Hernández, Lira et al., 2003, p. 27). Como resultado de la implementación de estas decisiones, en el transcurso del último quinquenio, cerca de un tercio de las carreras de grado que ofrece la institución, recibió el sello de calidad otorgado por el Sistema Nacional de Acreditación de la Educación Superior (SINAES) y por 


\begin{abstract}
This paper describes the development and innovation in the curriculum design of the Agricultural Engineering undergraduate program of the Technological Institute of Costa Rica. After its 30 years of existence, this program has reached its maturity. However, due to the changes in the development of agriculture, national and international tendencies, as well as the productive surroundings of the area and the social and academic context of the university, it faces the necessity to modify and innovate its curriculum. The main objective of the process consists of improving the quality in this area, proposing an integral redesign of the program. The antecedents, the methodology, and the most outstanding achievements of the process are described in this paper.
\end{abstract}

Key Words: Curriculum Design; Agricultural Engineering; Engineering Education; Higher Education. el Consejo Canadiense de Acreditación de Programas de Ingeniería (CEAB).

También es notorio que específicamente los programas de ingeniería del sector agro ofrecidos por el TEC (Ingeniería Agropecuaria Administrativa, Ingeniería en Agronomía e Ingeniería Agrícola), con la asesoría del Centro de Desarrollo Académico (CEDA), fueron colocados en la vanguardia del proceso de automejoramiento. A sabiendas de que la renovación y el fortalecimiento de la agricultura en Costa Rica es un tema complejo, sensible y de interés nacional (Antillón, Chang, Gámez et al., 2005, p.59), es de gran importancia que las carreras relacionadas directamente con ese ámbito generen procesos profundos e integrales de revisión, actualización e innovación de sus planes de estudio.

Como ejemplo de la evolución de la oferta académica del ITCR, se puede citar la autoevaluación y rediseño integral del plan de estudios realizado por la licenciatura en Ingeniería Agrícola en conmemoración de su 30 aniversario. Este hecho le permite asumir un compromiso renovado con los estudiantes y el país, además de ofrecer un programa formativo respaldado no solo por varias décadas de tradición, sino también por la consideración y reconocimiento de las tendencias socio-económicas, disciplinarias, curriculares y pedagógicas emergentes. A continuación, se describen algunos de los rasgos más prominentes del rediseño: sus antecedentes, la metodología empleada y los principales logros del proceso.

\section{Antecedentes}

La situación del desarrollo regional y mundial conduce a la inminente apertura de todos los sectores y a una agresiva competencia por la colocación de sus productos y servicios en el mercado, lo que exige las mejores condiciones para la producción. En el caso del sector agropecuario, la dependencia de múltiples variables de carácter natural, acentúa la necesidad de ser altamente eficiente en los procesos productivos. 
Por otra parte, "El desarrollo económico y social de nuestro país ha estado ligado a la producción agrícola como actividad generadora de fuentes de trabajo, riqueza y divisas" (Arias, 2005, p. 2). En consecuencia, ahora la presión se orienta hacia el mejoramiento sustancial de los aspectos como los derechos laborales, la certificación agrícola, el manejo ambiental y la optimización de los recursos. Estos elementos no solo otorgan valor agregado a los productos, sino también permiten emprender acciones para conservar los recursos naturales y coadyuvar con el desarrollo social.

En este contexto complejo se perfila y se valora el rol de la Ingeniería Agrícola, cuyo objeto de trabajo consiste en la generación de obras rurales relacionadas con la producción sostenible, desde su concepción, diseño, construcción, selección y planificación, hasta la administración y mantenimiento. Todo esto con el fin de mejorar las condiciones agro-socioeconómicas y mitigar la posibilidad de escenarios adversos que puede presentar la naturaleza, así como facilitar el trabajo de la población relacionada y aumentar la producción para beneficio de la sociedad en general.

Actualmente los graduados de esta carrera laboran en instituciones estatales (como municipalidades, Instituto Costarricense de Electricidad, Ministerio de Agricultura y Ganadería, Servicio Nacional de Riego y Avenamiento, Instituto Nacional de Aprendizaje, Instituto Costarricense de Acueductos y Alcantarillados), en el sistema bancario nacional, colegios técnicos profesionales, universidades, empresas productoras (como compañías de cultivos intensivos para la exportación y dedicadas a productos no tradicionales) y compañías comercializadoras de equipo especializado para la solución de problemas técnicos en las fincas productoras (como sistemas de riego y maquinaria agrícola). También se desarrollan como empresarios independientes y asesores de proyectos en las distintas áreas de la ingeniería agrícola.

La escuela de Ingeniería Agrícola, busca fortalecer su respuesta al contexto planteado mediante el rediseño de la carrera, gracias a un plan de estudios que permite la formación de profesionales calificados que generan, adaptan e incorporan la tecnología necesaria para el aprovechamiento de recursos y fuerzas productivas, contribuyendo así al desarrollo del país y a la transformación de la sociedad costarricense.

La continua búsqueda de la calidad abarca todos los elementos involucrados en la formación de profesionales en esta disciplina. La escuela de Ingeniería Agrícola está consciente de la necesidad de ampliar su horizonte con una visión de liderazgo hacia el uso de tecnologías actualizadas, por ejemplo, el diseño, construcción y manejo de estructuras para protección de cultivos, la utilización de sistemas de información geográfica, el desarrollo de software especializado, así como en el desarrollo de nuevas competencias de comunicación en el idioma inglés, la estimulación de la habilidad para liderar y gerenciar, el reforzamiento de la investigación y la motivación para el emprendedurismo, entre otras.

Para la adecuada integración de todas estas variables el rediseño de la carrera requiere de una metodología congruente.

\section{Metodología}

El carácter planificado, legal, equilibrado, prospectivo, liderado, participativo y concensuado son algunos de los rasgos más prominentes de la metodología del rediseño curricular de la licenciatura en Ingeniería Agrícola.

\subsection{Carácter planificado y legal}

La redefinición del programa, cuyo matiz es el desarrollo e innovación, constituye un reto complejo y alcanzable a mediano plazo. En este caso, la labor empezó en julio del 2005 y finalizó en noviembre del 2006. Desde su principio, este trabajo, implicado en un proceso más amplio de autoevaluación y automejoramiento de la carrera, contó con un mapa de ruta (figura $\mathrm{N}^{\circ} 1$ ) que guiaba todo el esfuerzo a través de este periodo. 


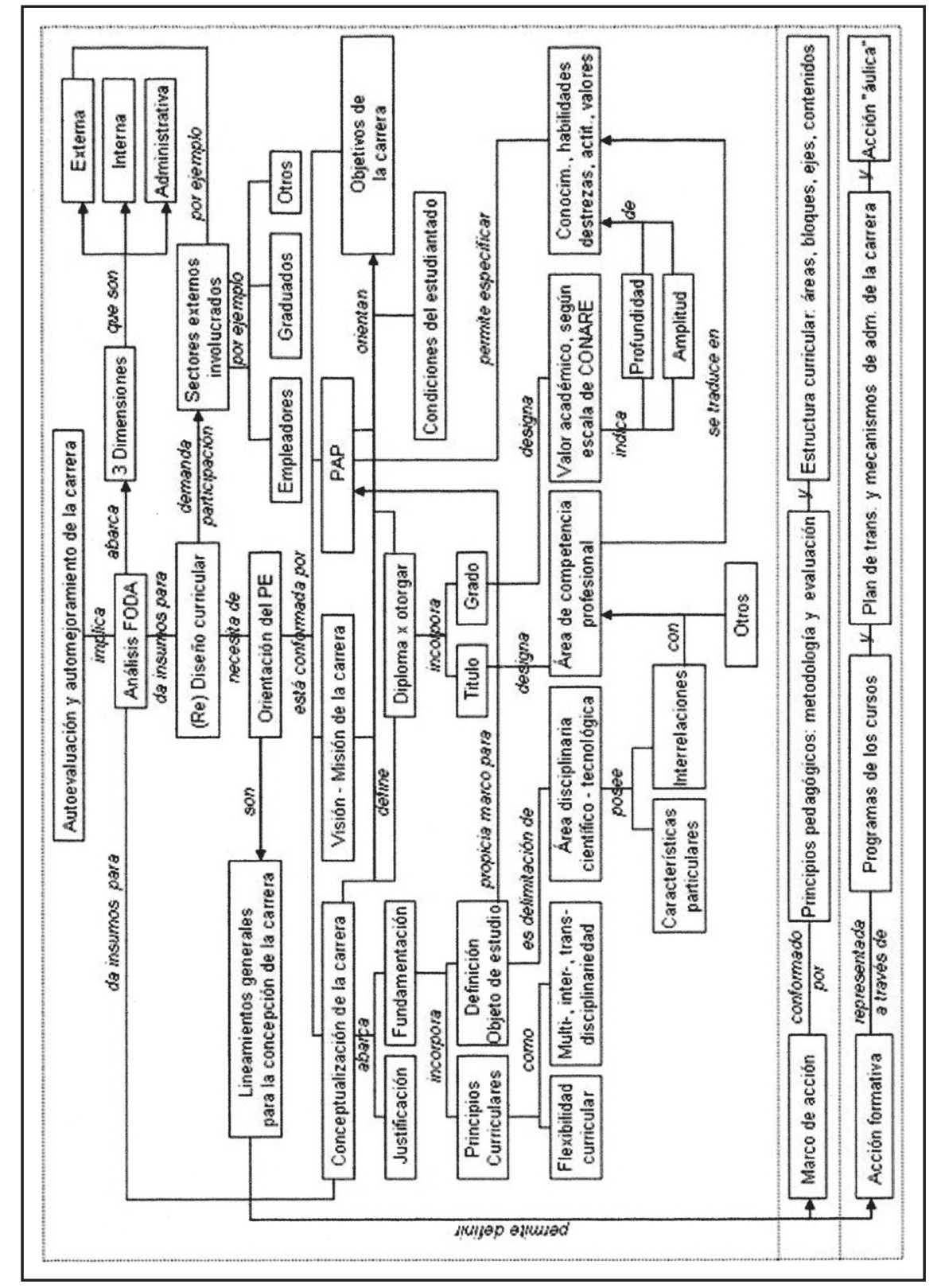

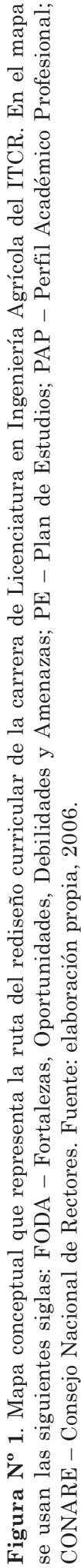


La existencia de un mapa de ruta le da al rediseño un carácter sistemático, pues ubica cada elemento en función de su relación con la totalidad y permite efectuar y ajustar los planeamientos de cada etapa en forma congruente.

El mapa se construyó a partir de un análisis previo de un amplio, pero selecto espectro de fuentes académico-administrativas que orientan y normalizan el desarrollo curricular en la institución. Para tal efecto se aprovechó el Manual para el diseno de planes de estudio y actualizaciones curriculares (ITCR, 2000), el Modelo académico del Instituto Tecnológico de Costa Rica (ITCR, 2003), la Guía metodológica sobre el diseño curricular en los planes de estudio (Quesada, Cedeño y Zamora, 2001), el Fluxograma para la creación de nuevas carreras o la modificación de carreras ya existentes (CONARE, 2004a), los Indicadores para medir la incorporación de la dimensión ambiental en los currícula de las Universidades Estatales de Costa Rica (CONARE, 2004b), la Guía para elaborar y autoevaluar el programa del curso (Fëdorov, 2004) y las Directrices curriculares para carreras de Ingeniería en Iberoamérica (ASIBEI, 2005), entre otros.

En este mismo apartado es importante resaltar la inscripción del proceso de desarrollo e innovación curricular del programa dentro de una gran meta: el mejoramiento de la calidad y su acreditación internacional. Por tanto, los elementos del mapa, sobre todo los relacionados con la estructura curricular y las competencias de los graduados, han sido pensados considerando los referentes aportados por los organismos nacionales e internacionales que acreditan los programas en el área de ingeniería. Entre estos indicadores y estándares destacan los aportados por la Agencia de Acreditación de los Programas de Ingeniería y de Arquitectura (CFIA, 2004) de Costa Rica, el Sistema Nacional de Acreditación de la Educación Superior (SINAES, 2000), el Consejo de Acreditación de la Enseñanza de la Ingeniería (CACEI, s.f.) de México, el Consejo de Acreditación de los Programas de Ingeniería y Tecnología (ABET, 2004) de los Estados Unidos, el Consejo Canadiense de Acreditación de los Programas de Ingeniería (CEAB, 2004), el Consejo Nipón de Acreditación de la Educación en Ingeniería (JABEE, 2002) y de la Agencia Registrada de Acreditación de Estudios de Ingeniería e Informática (ASIIN, s.f.) de Alemania, entre otras. La correspondencia del rediseño con estas orientaciones y normativas le otorga al proceso el debido perfil legal y además, en perspectiva, faculta el programa renovado a ser sujeto a una certificación internacional de su calidad.

\subsection{Carácter equilibrado y prospectivo}

También se afirma que la renovación curricular es un proceso equilibrado y prospectivo, ya que en calidad de cimientos toma todas aquellas buenas tradiciones y ventajas que el programa ha adquirido en el transcurso de los 30 años de su existencia. Por ejemplo, se puede mencionar que al rediseñar la estructura curricular de la carrera de la licenciatura en Ingeniería Agrícola del ITCR, se respetaron las fortalezas formativas con las que ya se cuenta en las áreas de las Ciencias Básicas, Ciencias de la Ingeniería y Manejo y Conservación de los Recursos Agua y Suelo.

No obstante, debido al espíritu de desarrollo e innovación del proceso, las buenas prácticas del pasado se ven a través del prisma de las tendencias actuales en el ámbito político, socioeconómico, disciplinar, curricular y pedagógico. De este modo, el nuevo plan de estudios, tanto en los contenidos de sus asignaturas como en la metodología del aprendizaje y la enseñanza, considera las implicaciones de la diversificación, crecimiento, modernización y apertura comercial del sector agro en el país. Por esta razón, se concentra en el reforzamiento de los aspectos relativos 
al emprendedurismo, la responsabilidad social y ambiental, investigación, gestión, normativas, comunicación, administración, mantenimiento de maquinaria y desarrollo de las tecnologías para la producción en la formación de un ingeniero agrícola de nueva generación.

Por supuesto, a la hora del análisis respectivo, los trabajos en el campo relacionado con el sector agro y la enseñanza de la ingeniería han sido de mucha ayuda. Las fuentes editadas por la Organización de las Naciones Unidas para la Educación, la Ciencia y la Cultura (UNESCO, 1998), la Organización Internacional de Trabajo (OIT, s.f.), la Asociación Iberoamericana de Instituciones de la Enseñanza de la Ingeniería (ASIBEI, 2005), el Consejo Superior Universitario Centroamericano (CSUCA, s.f.), el Proyecto Tuning (González y Wagenaar, 2003), el Proyecto 6 x 4 UEALC, al igual que los escritos de algunos autores nacionales y latinoamericanos (Arredondo, s.f.; Gómez y Celis, 2004; Lechuga, 2002; Márquez y Marquina, s.f.; Pereyra, 2000; Posada, s.f.; Pulido, s.f.; Soto, 1993; Tünnerman, 2000), entre otros, han servido como insumos de gran valor.

Asimismo, se consideró la oferta curricular nacional complementaria del programa homónimo de la Universidad de Costa Rica (UCR, 1998). Esta carrera, en el área de diseño de ingeniería, enfatiza los aspectos de manejo postcosecha y la carrera del ITCR tradicionalmente se centra más en el proceso productivo.

\subsection{Carácter liderado}

Por otro lado, se afirma que la redefinición del programa nace de un proceso liderado, ya que ha estado a cargo de una Comisión Coordinadora, la cual fue integrada por seis personas: el director de la escuela, dos docentes, un administrativo, un estudiante y un asesor del Centro de Desarrollo Académico (CEDA). Uno de los docentes se desempeñó como coordinador de la Comisión y, durante el último año del proceso, se le asignaron 20 horas semanales dentro de su carga laboral para el desempeño de dicha función. Otro profesor disponía de 8 horas semanales para realizar los trabajos relacionados.

En complemento y como ocurre en casi todos los procesos similares efectuados por el ITCR, la Comisión Coordinadora recibió apoyo de un funcionario del CEDA, al cual se le asignaron 8 horas semanales para asumir este compromiso. El asesoramiento curricular y pedagógico brindado por este departamento, concebido para apoyar la docencia del ITCR, supone un total involucramiento y un acompañamiento permanente. Sin embargo, este no es directivo, ya que las responsabilidades por las decisiones tomadas son plenamente asumidas por la Comisión encargada del rediseño y por el Consejo de la Escuela ya mencionada. Por lo tanto, el rol del asesor parte de la sistematización de la teoría y las experiencias de calidad que se acumulan en el CEDA y consiste en mapear los caminos a seguir, mostrar las posibles alternativas, sus implicaciones, alcances, ventajas y desventajas, procurar la inteligencia curricular y el apoyo metodológico para la comisión y la escuela que emprende el proceso de autoevaluación y rediseño.

Con el fin de alcanzar exitosamente la culminación de este proceso, es preciso generar y modular fuerzas de cohesión proyectadas hacia los sectores internos y externos a la unidad académica e institución, lo cual supone una participación muy activa de la dirección y una fuerte capacidad de liderazgo por parte de la Comisión Coordinadora.

\subsection{Carácter participativo y consensuado}

Otra de las cualidades del proceso es su carácter participativo y consensuado. La metodología del rediseño valida y combina la información obtenida de las fuentes bibliográficas y los aportes generados 
durante los talleres y reuniones de trabajo realizados con la participación de los académicos de la escuela de Ingeniería Agrícola, los representantes de los departamentos académicos de servicio, los estudiantes de la carrera, los graduados, los empresarios y empleadores del área agrícola.

Por ejemplo, como parte del proceso de rediseño, con los aportes de los sectores mencionados, guiados por la Comisión Coordinadora, se ha realizado una valoración del contexto nacional e internacional en el que se insertan los graduados de la carrera. Este análisis reveló la necesidad de reforzar los aspectos del programa relacionados con la responsabilidad social y ambiental, la promoción del desarrollo en las zonas rurales, la actualización permanente ante la dinámica e internacionalización de los mercados, entre otras cosas. Estas conclusiones llevaron a reforzar los elementos curriculares y pedagógicos coadyuvantes con el desarrollo de las competencias emergentes en las áreas de formación humanística, comunicación, emprendedurismo, gestión, normativas, mecanización y desarrollo y adaptación de tecnologías para la producción.

Como ejemplo del carácter representativo del proceso, se puede mencionar el involucramiento de los graduados y de los empleadores. El interés y los aportes de los delegados de las empresas e instituciones dedicadas a riego (Durman Esquivel, Irrigación de Costa Rica, Amanco, Riegos Modernos), administración y mantenimiento de maquinaria agrícola (Instituto Nacional de Aprendizaje, Macori S.A., Hacienda Juan Viñas) y de desarrollo y adaptación de tecnologías para la producción (Linda Vista, Melones de Costa Rica), entre otras, han sido muy significativos para redefinir el perfil académico profesional e innovar en la estructura del plan de estudios, para reforzar los elementos curriculares relacionados con la administración, la investigación, el inglés, el desarrollo de las tecnologías para la producción y su adaptación.
La ruta del rediseño fue marcada por una serie de puntos cruciales, en los cuales se conjugaban distintos sectores interesados. Estos momentos de comunión de los actores del proceso de rediseño permitían perfilar el camino a seguir de manera participativa y consensuada. Como un ejemplo más que ilustra el carácter democrático de todo el proceso, se puede mencionar el taller denominado "Hacia una meta inspiradora, precisa y compartida", en el cual se inició el examen de la visión y la misión de la carrera. Esta actividad contó con la participación de todo el personal docente y administrativo y con la representación de los estudiantes, graduados y empleadores de la carrera. De igual modo, los objetivos de la carrera se elaboraron a partir del taller denominado ¿A dónde se dirigen nuestras acciones?; los principios curriculares se redefinieron a partir de un taller, llamado Construyendo sobre bases sólidas - Ideas fundamentales que rigen el plan de estudios, seguido de varias sesiones de trabajo durante tres semanas. El rediseño del perfil académico profesional del licenciado en Ingeniería Agrícola demandó una importante inversión de tiempo y la meta de su reelaboración se alcanzó durante el taller llamado Egresado de la Licenciatura en Ingeniería Agrícola del ITCR: un profesional competente, competitivo y comprometido.

En confirmación del carácter legal y consensuado del proceso, cada uno de los productos intermedios del rediseño de la carrera (la visión y la misión del programa, su objeto de estudio, la fundamentación y justificación, los principios curriculares, el perfil académico profesional, los objetivos, la estructura curricular, los principios metodológicos y evaluativos, los programas de los cursos, el plan de transición, el sistema de equivalencias, el plan de divulgación y atracción) fueron aprobados paulatinamente por la Comisión Coordinadora y el respectivo Consejo de Escuela.

Una vez finalizado el proceso, el informe completo fue sometido al análisis 
técnico curricular por parte del Centro de Desarrollo Académico y éste lo dictaminó positivamente. Y, para culminar, en noviembre del 2006, el rediseño curricular de la carrera de licenciatura en Ingeniería Agrícola del ITCR fue presentado y aprobado por el Consejo de Docencia, el cual está compuesto por los representantes directivos de todos los departamentos académicos de la institución.

Como discurre de la breve descripción anterior, la metodología empleada para llevar a cabo el rediseño curricular del programa, al estar sustentada por los recursos necesarios, permitió integrar y aprovechar los aportes de diferentes fuentes y actores sociales, profesionales y académicos, los cuales bajo el liderazgo de una Comisión Coordinadora, el asesoramiento del CEDA y la venia del Consejo de Escuela y de Docencia, lograron producir una propuesta formativa renovada, pertinente, legal y consensuada en un tiempo prudente.

\section{Logros}

Las características descritas en el apartado anterior de este rediseño, evidencian la calidad con la que se implementó una serie de modificaciones, producto de un proceso altamente reflexivo y contextualizado a la realidad del entorno de la carrera y la institución a la que pertenece.

El primero de los productos obtenidos fue la actualización de la visión y la misión de la carrera. Se logró la redefinición de las áreas específicas que constituyen el plan de estudios y el compromiso de mantener el nivel de desempeño exigido para el fortalecimiento de la docencia, la investigación y la extensión. Así, estos dos elementos del rediseño orientaron la revisión, la concepción y maduración de los demás frutos obtenidos.

Dentro de la organización de insumos que dieron pie a las siguientes etapas se hizo un análisis de las fortalezas, debilidades y amenazas para ubicar las oportunidades de mejora del programa, lo cual dio origen a una sólida justificación del proceso. Estos pasos, a su vez, permitieron redefinir con claridad el objeto de estudio, especificar las áreas y subáreas de la disciplina e incorporar los ejes transversales de la carrera. Por otra parte, se espera que: "El objeto de estudio se nutra aún más de la investigación, resultados de la cual se transfieran eficientemente a los estudiantes y a los productores". (ITCR-IA, 2006, p. 6)

Posteriormente se oficializaron diez principios curriculares para la definición de los elementos operativos del plan de estudio renovado. Estos principios son los siguientes: secuencia vertical, coherencia horizontal, continuidad, integración disciplinar (en su expresión de multi e interdisciplinariedad), flexibilidad, identidad, vinculación y pertinencia social y académica, universalidad, internacionalización y transversalidad. Este último principio abarca los temas de calidad, emprendedurismo, liderazgo, civismo, pensamiento crítico, adaptabilidad, investigación, manejo de información, trabajo en equipo, comunicación, dimensión ambiental y sostenibilidad, seguridad y salud integral.

Otro de los logros más destacados fue el rediseño del perfil académico profesional, para el cual se utilizó un modelo que combina las competencias con los aprendizajes fundamentales, integrando así las tres principales áreas de formación: la personal-ciudadana, la académica-ingenieril y la profesional-laboral.

También se fijaron los nuevos objetivos de la carrera que permitirán mantener un horizonte y avanzar permanentemente hacia la condición definida en los elementos orientadores del plan de estudio. Entre estos objetivos se puede mencionar, por ejemplo: "Mantener un análisis constante del entorno para poder responder a la demanda del medio, referente a la actualización del personal y del plan de estudios, nuevas tecnologías y acciones de apoyo al sector productivo". (ITCR-IA, 2006, p. 27) 
Asimismo, se redefinió la estructura curricular derivada de las ochograndesáreas que componen el plan de estudios: Ciencias Básicas, Ciencias Sociales y Económicas, Ciencias de la Computación, Ciencias de la Ingeniería, Manejo y Conservación de los Recursos Agua y Suelo, Administración y Mantenimiento de Maquinaria Agrícola, Desarrollo y Adaptación de Tecnologías para la Producción y Trabajo Final de Graduación. A la hora del rediseño de esta estructura se buscó una mayor congruencia con otros elementos curriculares. En vista de lo anterior, se redistribuyó el peso académico por área y se reestructuró el diagrama del plan de estudios con la eliminación, creación y modificación pertinente de los cursos.

De la figura $\mathrm{N}^{\circ} 2$ se desprende que las mayores modificaciones, realizadas a partir del análisis de las tendencias y perspectivas del desarrollo del sector y de la disciplina a nivel nacional e internacional, principalmente afectan cuatro de las ocho áreas que conforman el plan de estudios de la carrera. En el caso de Ciencias de la Ingeniería (CI) y Manejo y Conservación de los Recursos Agua y Suelo
(MCRAS), su presencia relativa tiende a disminuir. Mientras que el peso formativo de Ciencias Sociales y Económicas (CSE) y Desarrollo y Adaptación de Tecnologías para la Producción (DATP) aumenta. También existe un incremento en el número de créditos correspondientes al Trabajo Final de Graduación (TFG).

Otro de los frutos del rediseño del plan de estudios fue conseguir una mayor flexibilidad. El curriculum rediseñado incluye tres asignaturas electivas de manera que el estudiante pueda escogerlas de un amplio menú de materias del área que más le llame la atención. Por ejemplo, de la oferta del área de Desarrollo y Adaptación de Tecnologías para la Producción se puede elegir cursos como Protección Ambiental y Tratamiento de Aguas Residuales, Manejo de Cultivos en Invernadero, Certificación en Agricultura o Sistemas de Información Geográfica.

Al final, todos los elementos orientadores, incluyendo los principios metodológicos y evaluativos, se operacionalizan a través de los programas de cada una de las 63 asignaturas del plan de estudio, listas para ser llevadas a la práctica.

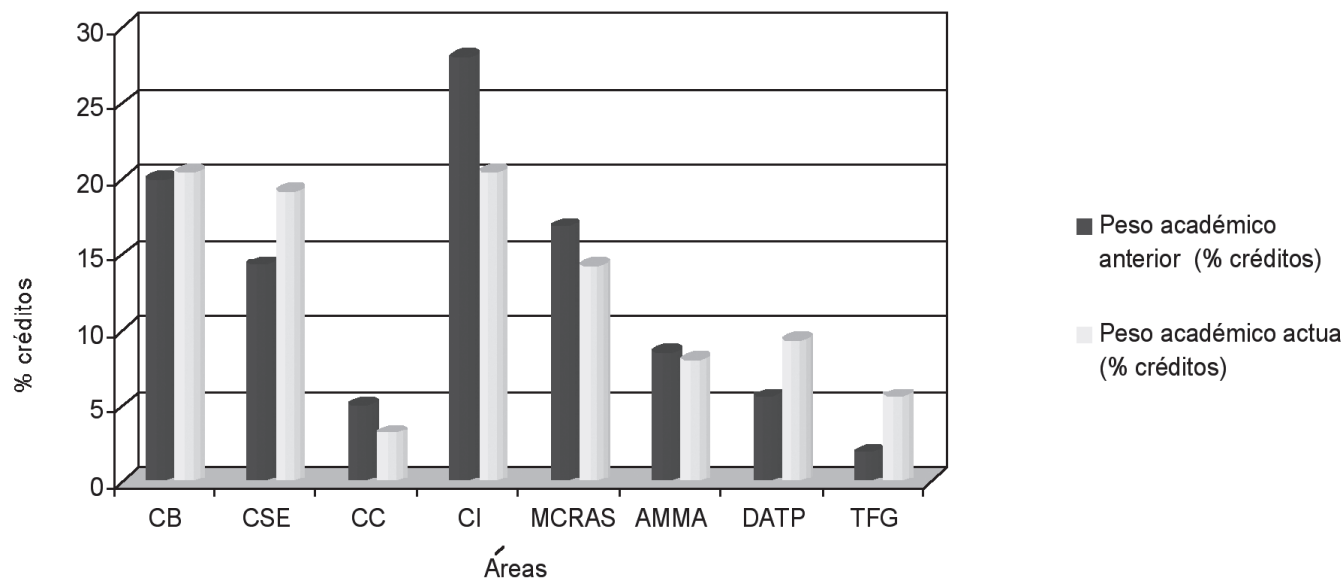

Figura $\mathbf{N}^{\mathbf{0}}$ 2. Gráfico de variación de los porcentajes de créditos correspondientes a cada área de la carrera. En el gráfico se usan las siguientes siglas: CB: Ciencias Básicas; CSE: Ciencias Sociales y Económicas; CC: Ciencias de la Computación; CI: Ciencias de la Ingeniería; MCRAS: Manejo y Conservación de los Recursos Agua y Suelo; AMMA: Administración y Mantenimiento de Maquinaria Agrícola; DATP: Desarrollo y adaptación de Tecnologías para la Producción y TFG: Trabajo Final de Graduación.

Fuente: elaboración propia, 2006. 
La puesta en marcha de todas estas modificaciones y su permanente evaluación se han tomado en cuenta para establecer un modelo de administración de la carrera orientado a conseguir el éxito del replanteamiento de la Ingeniería Agrícola en el ITCR. Ha sido necesario, por lo tanto, establecer un programa de transición para los estudiantes regulares con el fin de ir implementando de manera paulatina el rediseño de la carrera. También ha sido fundamental crear un plan de divulgación que muestre el nuevo enfoque de la oferta curricular a la población meta de esta importante disciplina.

\section{Conclusiones}

En conclusión, se puede afirmar que gracias a un trabajo participativo, coordinado, consensuado y sustentado por una metodología que combina las asignaciones, las responsabilidades, los controles, las evaluaciones y la toma de decisiones tanto en forma individual como grupal, que considera las tendencias curriculares, pedagógicas y disciplinarias contemporáneas, se ha logrado rediseñar un plan de estudios de la carrera de Ingeniería Agrícola de manera integral en un tiempo prudente.

Este plan de estudios modificado, que suma 157 créditos distribuidos en 10 períodos lectivos, logra una mayor consistencia de los elementos que lo conforman e incluye 62 cursos semestrales, Centros de Formación Humanística y un trabajo final de graduación de un año de duración para culminar.

La renovada carrera de Ingeniería Agrícola del ITCR abre sus puertas a partir del I semestre del 2007 y, gracias a un efectivo plan de promoción, ya cuenta con un aproximado de cuarenta estudiantes matriculados.

La escuela de Ingeniería Agrícola del ITCR y el CEDA, concientes de la importancia del análisis crítico y constante del entorno académico y disciplinar, invitan a los colegas de otras universidades y sectores interesados a compartir sus ideas sobre los temas de desarrollo e innovación en los planes de estudio de los programas de ingeniería del sector agro, con el fin de lograr una mejor calidad y competitividad de las sociedades latinoamericanas. Pueden enviar sus comentarios a los correos electrónicos iguzman@itcr.ac.cr y afedorov@ itcr.ac.cr, y con gusto los atenderemos.

\section{Referencias bibliográficas}

Accreditation Board for Engineering and Technology, ABET. (2004). Criteria for Accrediting Engineering Programs Effective for Evaluations during the 2005-2006 Accreditation Cycle. Engineering Accreditation Commission. ABET Inc. Baltimore, MD. Recuperado el 12/12/05 del sitio Web del ABET en http://www.abet.org

Agencia Registrada de Acreditación de Estudios de Ingeniería e Informática, ASIIN. (s.f.). Información dirigida al mundo universitario. Requisitos y principios para la realización de estudios de ingeniería e informática a nivel de Bachelor y Máster. Recuperado del sito Web del ASIIN en http://www.asiin.de

Antillón, A., Chang, F., Gámez, R., et al. (2005). Informe final de carácter general no vinculante al Presidente de la República sobre el Tratado de Libre Comercio entre Centroamérica, República Dominicana y Estados Unidos de América. Cartago, Costa Rica: Editorial Tecnológica de Costa Rica.

Arias, O. (2005). Retos para la agricultura en Costa Rica. Agronomía Costarricense, 29 (2), 157-166. Disponible en el sitio Web de la versión digital de la revista en: 
http://www.mag.go.cr/rev_agr/ v29n02 157.pdf

Arredondo, V. (s.f.). Políticas de flexibilidad curricular en educación superior. Recuperado 22/01/2006 del sitio Web de la Universidad Autónoma de Tamaulipas, México en http://colaboracion.uat.edu.mx/ rectoria/subacademica/documentos/ polflexcurr.pdf

Asociación Iberoamericana de Instituciones de la Enseñanza de la Ingeniería, ASIBEI. (2005). Directrices curriculares para carreras de Ingeniería en Iberoamérica. Bogotá, Colombia: ARFO Ediciones e Impresiones Ltda.

Consejo de de Acreditación de la Enseñanza de la Ingeniería, CACEI. (s.f.). Manual del CACEI. Recuperado del sitio Web del CACEI el 12/12/2005 en http://www.cacei.org/index.html

Canadian Engineering Accreditation Board, CEAB. (2004). Accreditation Criteria and Procedures. Recuperado 24/06/2005 del sitio Web del Canadian Council of Professional Engineers en http://www.ccpe.ca/e/acc_supp_1.cfm

Colegio Federado de Ingenieros y de Arquitectos de Costa Rica, CFIA. (2004). Criterios y procedimientos de acreditación: Programas de ingeniería, 2004. San José, C.R.: CFIA, Departamento de Formación Profesional.

Consejo Nacional de Rectores, CONARE. (2004a). Fluxograma para la creación de nuevas carreras o la modificación de carreras ya existentes. San José (C.R.): CONARE, OPES publicaciones.

Consejo Nacional de Rectores, CONARE. (2004b). Indicadores para medir la incorporación de la dimensión ambiental en los currícula de las Universidades Estatales de Costa Rica. San José, C.R.: CONARE, Comisión Interuniversitaria de Educación Ambiental.

Consejo Superior Universitario Centroamericano, CSUCA. (s.f.). Promedios de estructura curricular por universidades: cuadro comparativo de la oferta académica en horas obligatorias por universidad. Archivo personal A. Fëdorov.

Fëdorov, A., Hernández, F., Lira, R., et al. (2003). Diagnóstico institucional sobre el estado de la gestión de los procesos de autoevaluación con propósitos de mejoramiento continuo y de acreditación, de las carreras del ITCR, a partir de 1998. Cartago, C.R.: Proyecto CAMINA 2003, ITCR, Centro de Desarrollo Académico, material inédito disponible en el archivo del centro.

Fëdorov, A. (2004). ¿Cómo programar un curso? Guía para elaborar y autoevaluar el programa del curso. Cartago, C.R.: Editorial Tecnológica de Costa Rica.

Gómez, V. y Celis, J. (2004). Factores de innovación curricular y académica en la educación superior. Documento Word recuperado 22/01/2006 del Sitio Web de la Universidad Nacional de Colombia en http://www.unal.edu.co/ documentos/Anexos_lineamientos curriculares.doc

González, J. y Wagenaar, R. (2003). Tuning Educational Structures in Europe: Informe Final Fase Uno. Bilbao: RGM, S.A.

Instituto Tecnológico de Costa Rica, ITCR. (2000). Manual para el diseño de 
planes de estudio y actualizaciones curriculares. Cartago, C.R.: ITCR, Vicerrectoría de Docencia, Centro de Desarrollo Académico.

Instituto Tecnológico de Costa Rica, ITCR. (2003). Tercer Congreso Institucional: Modelo académico del ITCR. Cartago, C.R.: ITCR, Taller de publicaciones.

Instituto Tecnológico de Costa Rica, ITCR. (2005). Estatuto Orgánico del Instituto Tecnológico de Costa Rica y sus reformas. Cartago, C.R.: Editorial Tecnológica de Costa Rica.

Instituto Tecnológico de Costa Rica, Escuela de Ingeniería Agrícola, ITCR-IA. (2006). Rediseño de la carrera de Licenciatura en Ingeniería Agrícola. Documento inédito, disponible en el archivo de la Escuela de la Ingeniería Agrícola.

Japan Accreditation Board for Engineering Education, JABEE. (2002). Criteria for Accrediting Japanese Engineering Education Programs 2002-2003. Documentopdfrecuperado 12/12/2005 del sitio Web del Japan Accreditation Board for Engineering Education En http://www.jabee.org/english

Lechuga, C. (2002). Evaluación y Acreditación de la Enseñanza de la Ingeniería en Centroamérica: Estudio de Diagnóstico de la Demanda y Oferta Académicas en los Mercados Laborales en el Sector de ingeniería. Guatemala: SP-CSUCA-REDICA.

Márquez, A. y Marquina, M. (s.f.). Evaluación, Acreditación, Reconocimiento de Títulos y Habilitación: Enfoque comparado. Documento pdf recuperado 30/12/2005 del sitio Web de la Comisión Nacional de Evaluación y Acreditación Universitaria, Argentina en http://www.coneau.edu.ar/que_es/ document/publicaciones/marquez marquina.pdf

Organización de las Naciones Unidas para la Educación, la Ciencia y la Cultura, UNESCO. (1998). Declaración mundial sobre la educación superior en el siglo XXI: visión y acción y marco de acción prioritaria para el cambio y el desarrollo de la educación superior. Documento html recuperado 12/10/2003 del sitio Web de UNESCO en: http://portal. unesco.org/education/es/ev.php$\mathrm{URL} \_\mathrm{ID}=7152 \& \mathrm{URL}_{-} \mathrm{DO}=\mathrm{DO}_{-}$ TOPIC\&URL_SECTION=201.html.

Organización Internacional de Trabajo, OIT. (s.f.) Competencia laboral. Consultado 25/10/2005 en http://www.ilo.org/public/spanish/ region/ampro/cinterfor/temas/ complab/xxxx/esp/i.htm

Pereyra, A. (2000). Integración regional y reformulación curricular: Una mirada a la enseñanza de las ciencias sociales en el MERCOSUR. Montevideo: ANEP, Consejo de educación secundaria. Recuperado 30/12/2005 del http://www.anep.edu.uy/ces/mercoint. $\underline{\mathrm{htm}}$

Posada, R. (s.f.). Formación superior basada en competencias, interdisciplinariedad y trabajo autónomo del estudiante. Facultad de Educación, Universidad del Atlántico, Colombia. http:// www.campus-oei.org

Pulido, H. (s.f.). Flexibilidad Curricular. Seminario Fortalecimiento de la capacidad académica de las instituciones de Educación Superior. Ministerio de Educación Nacional. Bogota, Colombia: s.e.

Sistema Nacional de Acreditación de la Educación Superior, SINAES. (2000). 
Manual de acreditación: Convocatoria del año 2000. San José, Costa Rica: SINAES - CONARE.

Soto, R. (1993). Propuesta para un modelo curricular flexible. Revista de la educación Superior, 85, 103-108. Recuperado 30/12/2005 del sitio Web de la Universidad Autónoma de Tamaulipas, México, en http:// rectoria.uat.edu.mx/planeacion/ modelodeestructura.pdf

Tünnermann, C. (2000). Una Nueva Política para la Educación Superior en América Latina y Caribe. Memorias del IV Encuentro de Estudios Prospectivos Región Andina: Sociedad, Educación y Desarrollo. Medellín, Colombia: ESUMER. Recuperado del sitio Web del Instituto de Estudios Prospectivos de Antioquia, en http://www.esumer. edu.co/prospectan.html

Quesada, M., Cedeño, M. y Zamora, J. (2001). El diseño curricular en los planes de estudio: aspectos teóricos y guía metodológica. Heredia (C.R.): EUNA.

Universidad de Costa Rica (UCR). (1998). Ingeniería Agrícola: Plan de estudios - Bachillerato y Licenciatura. Sistema de Aplicaciones Estudiantiles SAE: Módulo Planes de Estudio, consultado 30/08/2005 en

http://www.cea.ucr.ac.cr/ planes/SEDE\%20UNIVERSITA RIA\%20RODRIGO\%20FACIO/ AREA\%20DE\%20INGENIERIAS/ FACULTAD\%20DE\%20INGENIERIA/ ESCUELA\%20DE\%20INGENIERIA \%20AGRICOLA/BACH.\%20Y\%20LIC.\% 20EN\%20INGENIERIA\% 20AGRICOLA\%201998.pdf 
